\title{
Supernova Neutrino Detection with LHAASO-MD
}

\section{D.Liu, ${ }^{a}{ }^{*}$ J.Chang, ${ }^{c}$ S.Chen, ${ }^{d}$ H.Dai, ${ }^{c}$ C.Feng, ${ }^{a}$ B.Gao, ${ }^{c}$ G.Gong, ${ }^{d}$ M.Gu, ${ }^{c}$ F.Li, ${ }^{c}$ X.Ma, ${ }^{c}$ X.Wang, ${ }^{b}$ Z.Wang ${ }^{d}$ and X.Zuo ${ }^{c}$ on behalf of the LHAASO Collaboration}

(a complete list of authors can be found at the end of the proceedings)

${ }^{a}$ Institute of Frontier and Interdisciplinary Science, Shandong University, Qingdao 266237, Shandong, China

${ }^{b}$ National University of Defense Technology,

Changsha 410073, Hunan, China

${ }^{c}$ Institute of High Energy Physics, Chinese Academy of Sciences, Beijing 100049, China

${ }^{d}$ Tsinghua University, Beijing 100084, China

E-mail: dliu@sdu.edu.cn, wangxi@nudt.edu.cn, maxh@ihep.ac.cn, fengcf@sdu.edu.cn

The core-collapse supernova releases a tremendous number of neutrinos, which can provide insight into many research areas, including particle physics, astrophysics, nuclear physics, and cosmology. We can detect the signal through a positron produced from the inverse beta decay (IBD) interaction between the electron antineutrino and water. The Large High Altitude Air Shower Observatory[1] muon detector (LHAASO-MD) with 51-kton water can serve this purpose. The MD detectors have been designed to have a scattered layout as well as spatial uniformity. We hope to design a dedicated supernova trigger system in the data acquisition system to take advantage of these unique detector characteristics. The large numbers of MeV-scale supernova burst neutrinos can be observed from a collective rise in all photomultiplier rates on top of the dark noise. This system should effectively suppress the cosmic ray background, optimizes the neutrino detection sensitivity, and realizes the supernova neutrino detection by optimizing the online trigger, data acquisition, and offline data analysis at LHAASO. The trigger system is estimated to be fully sensitive to 1987A-type supernova bursts throughout most of the Milky Way and can eventually help LHAASO join the SuperNova Early Warning System (SNEWS).

$37^{\text {th }}$ International Cosmic Ray Conference (ICRC 2021)

July 12th - 23rd, 2021

Online - Berlin, Germany

\footnotetext{
${ }^{*}$ Presenter
} 


\section{Introduction}

On February 24, 1987, a neutrino burst, which signified the event as a type II core-collapse supernova in the case of SN 1987A in the Large Magellanic Cloud at a distance of roughly $50 \mathrm{kpc}$, was retrospectively detected by several detectors ([2]). SN1987A provides a unique opportunity to observe the neutrino emission from a supernova. Besides the Sun, SN1987A remains the only known one to be detected by its neutrino emissions, which could provide a large range of physical limits on neutrinos as well as the core-collapse supernova mechanism. A total of 24 neutrino events in the Kamiokande[3], IMB [4], and Baksan[5] underground experiments were recorded over a time interval of about 12 seconds. About half of the events came in the first second. Most of the events came in the first two seconds. Their individual energies range are from $10 \mathrm{MeV}$ to $50 \mathrm{MeV}$, which refers to the energy of secondary positrons produced by the captures of such neutrinos on protons.

Supernova neutrino burst event is rare. The burst occurs at the rate of only a few per century [6]. The detection of the neutrino burst from the next galactic supernova can provide an early warning for astronomers. The Supernova Early Warning System (SNEWS[7]) is to provide a World-wide alert of the supernova neutrino signal. SNEWS could also serve as a trigger for those experiments, which not able to be triggered by supernova signals by themselves. Extra interesting data could be saved in this case.

\section{Design of LHAASO-MD}

The Large High Altitude Air Shower Observatory (LHAASO[8]) is a new generation experiment, which includes 1171 muon detectors (MDs) for measuring the number density and the arrival time of extensive air shower (EAS) secondary muons. The MDs are in a triangular grid with a spacing of 30m in the central part of the array, which has a scattered layout and spatial uniformity. The design with a water Cherenkov detector underneath the soil is chosen for MDs. For each MD unit, a water bag with a diameter of $6.8 \mathrm{~m}$ and a depth of $1.2 \mathrm{~m}$ is used to enclose pure water. The liner reflectivity for the water bag is better than $95 \%$, and the water absorption length is designed to be longer than $50 \mathrm{~m}$. There is a $2.5 \mathrm{~m}$ thickness of the overburdened soil over the MD water bag. An 8-inch PMT sits at the top center of the water bag and looks downward through a highly transparent window into the water. For each MD unit, there are 0.044 kton water and a total of 51 kton water for all 1171 MD units, which could provide a huge target for neutrino detection. Each MD has a detection efficiency better than $95 \%$.

Supernova neutrino is detected by the inverse beta process(IBD) in most experiments. IBD process describes a neutrino scattering off a proton and producing a neutron and positron

$$
\text { IBD process : } v_{e}+p \rightarrow n+e^{+} \text {. }
$$

In this process, positron carries almost all the energy from the neutrino. The neutrino detection experiments can be divided into two categories. The first category experiments could do the IBD event reconstruction. In this case, those background events can be suppressed effectively while usually, the target mass is small. The second category experiments could only see the collective rise in all events rates on top of the dark noise. In this category, there are many background events while the target mass is huge. LHAASO-MD is the second one. Huge target mass means more IBD Events. The comparison between LHAASO-MD and other experiments could be found in Table 1 . 


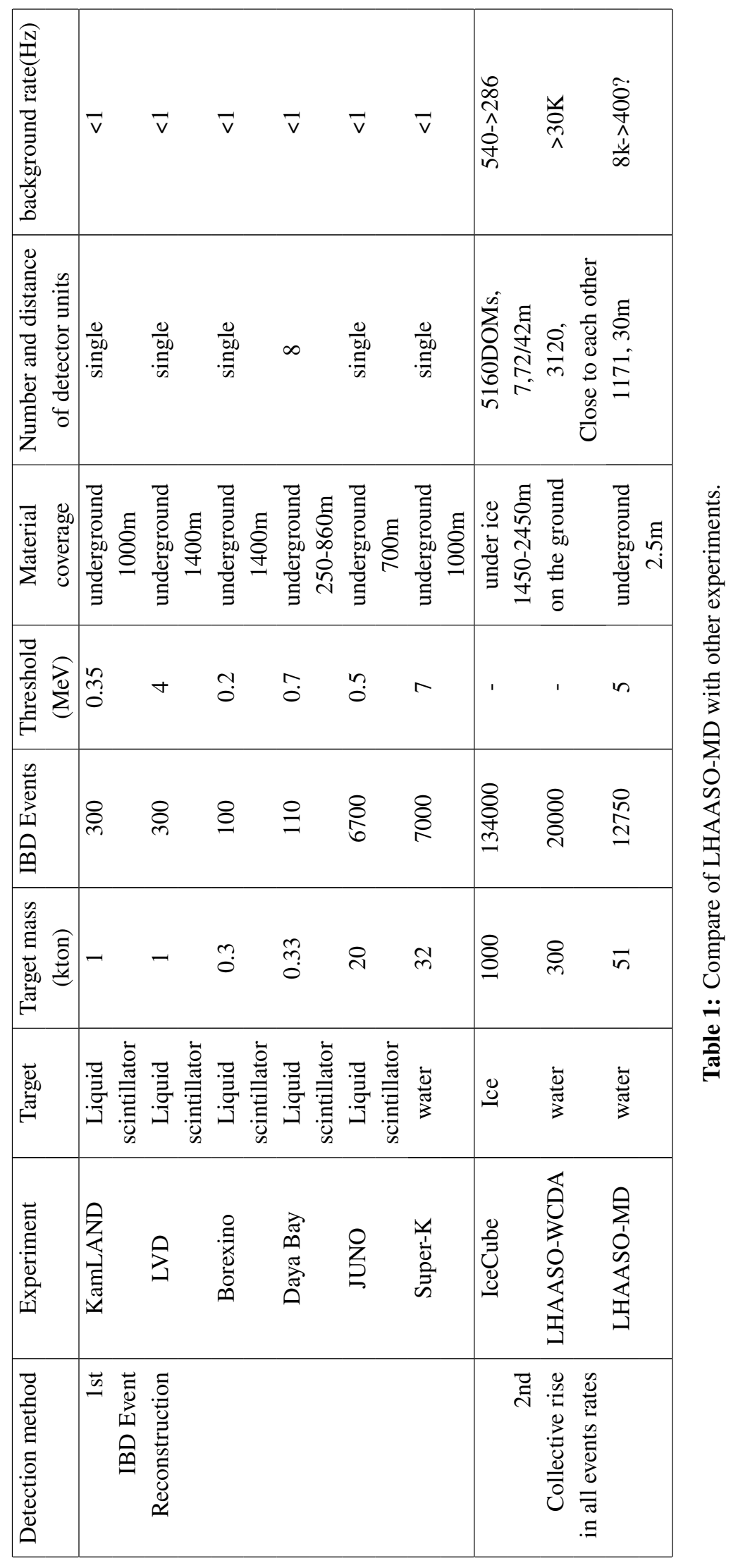




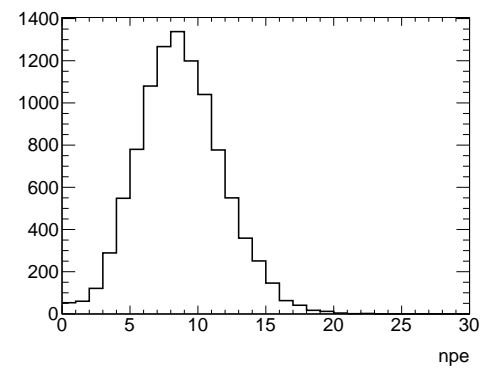

(a) Distribution of Photoelectron Number

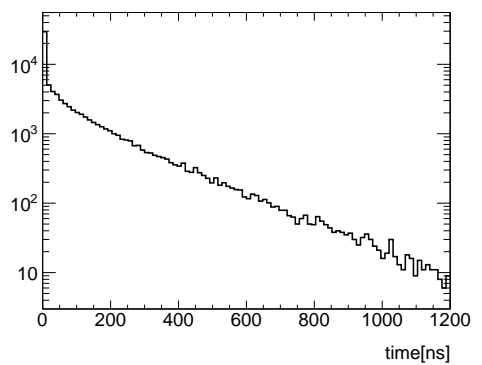

(b) Photoelectron arrival time for 10000 positron events. Time 0 is the positron injection time

Figure 1: The Monte Carlo simulation for $20 \mathrm{MeV}$ positron

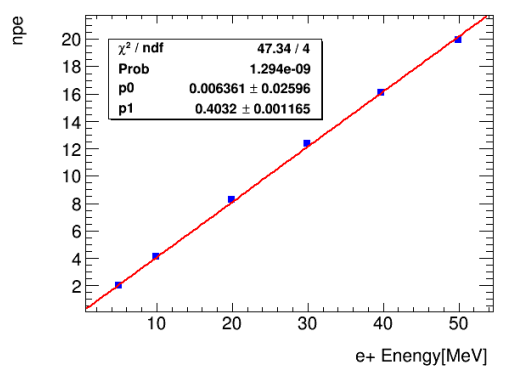

Figure 2: number of photoelectrons versus positron energy

\section{MD simulation and Detector performance}

10000 positrons are simulated and injected into MD detectors at different positions at energies of 5, 10, 20,30,40, and $50 \mathrm{MeV}$ by Geant4_10.4.2[9]. In Figure 1, the left plot shows the distribution of photoelectron numbers for $20 \mathrm{MeV}$ positrons. On average 8 photoelectrons will be collected for $20 \mathrm{MeV}$ positrons. The right plot shows the arrival time of the photoelectrons for $20 \mathrm{MeV}$ events. $97.2 \%$ of the photoelectrons arrive in $608 \mathrm{~ns}$. In the plot time $\mathrm{t}=0$ indicates the positron injection time. Figure 2 is the simulated number of photoelectrons versus positron energy. Photoelectron number increases with the positron energy. The figure could be fitted by:

$$
N_{\text {Photoelectron }}=0.4 \times E_{\text {Positron }}
$$

Here $N_{\text {Photoelectron }}$ denotes the expected photoelectron number from positron and $E_{\text {Positron }}$ is the injected positron energy. For $10 \mathrm{MeV}$ to $50 \mathrm{MeV}$ positrons from supernova neutrino, LHAASO-MD could receive 4 to 20 photoelectrons in hundreds of ns.

A special trigger mode with all MD hits recorded was tested on Sep 18, 2019, at LHAASO-MD. From those full recorded data, the single MD data acquisition rate is about $8000 \mathrm{~Hz}$. In Figure 3 the left plot is the read-out signal from a single MD. The first peak is the noise from electronics and other random sources, and the second one is from muon events. As the positron from neutrino events should be in the energy range from 10-50MeV[2]. Most noise and muon signals could be reduced by energy cut $10-100 \mathrm{MeV}(100-1000 \mathrm{ADC}$ counts). The right plot of Figure 3 shows the 


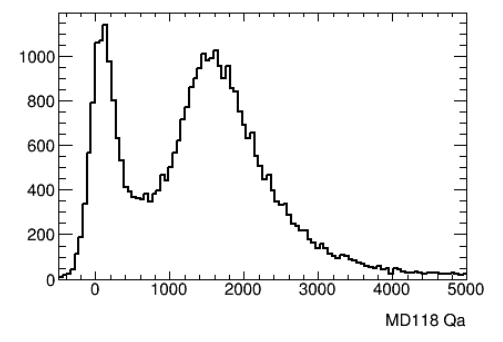

(a) Distribution of MD Qa

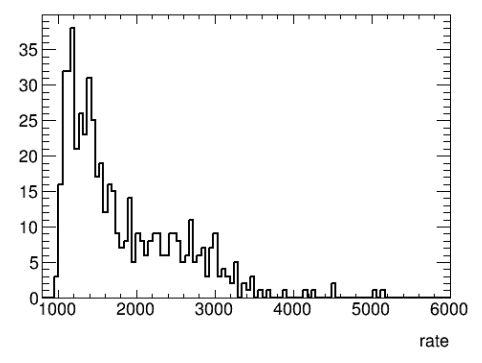

(b) Distribution of MD rate

Figure 3: Distribution of MD Qa and rate

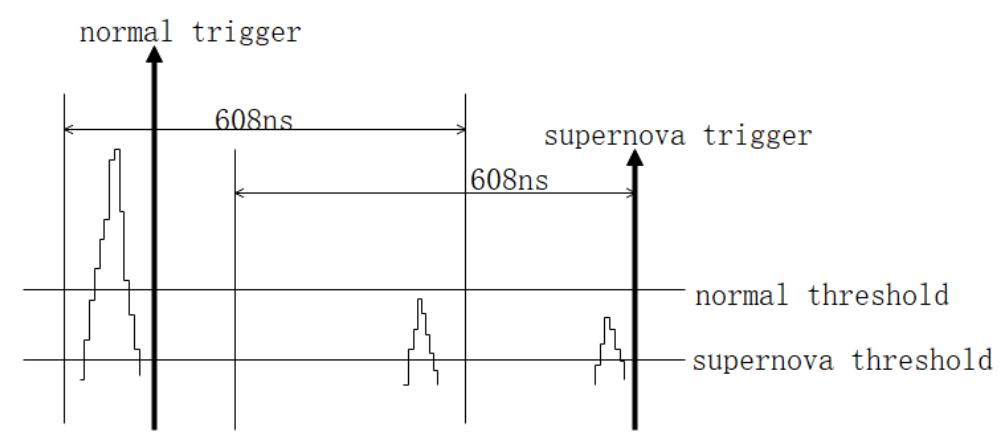

Figure 4: New trigger design for supernova neutrino

mean hits rate of 500 MDs between 100-1000ADC count. The average MD data rate is about 2000 $\mathrm{Hz}$ after the energy cut.

\section{FPGA programming}

For each MD, about 4-20 photoelectrons arriving in hundreds of ns for supernova neutrino signal. Normally there will be only about 1-2 photoelectrons arriving at the same time. The LHAASO normal MD threshold is 1.7 pe, which means at least two photoelectrons arriving in 2 ns will be triggered efficiently by the current setting. The trigger efficiency for neutrino from the supernova is low. A new trigger design is needed to trigger 4-20 photoelectrons in hundreds of ns.

The FPGA Level trigger programming with the sliding windows method is on progressing to trigger the neutrino events. This supernova trigger mode can be updated through the FPGA firmware in the MD electronics. The major idea of trigger designing is to find the neutrino events in the sliding windows of width 608ns. As Figure 4 shows, if many small signals in the sliding window exceed the supernova threshold, a supernova trigger will be sent out. The number of the small signals can be configured. This trigger mode will not affect the normal trigger mode as Figure 5 .

We hope this FPGA programming work could be done soon. 


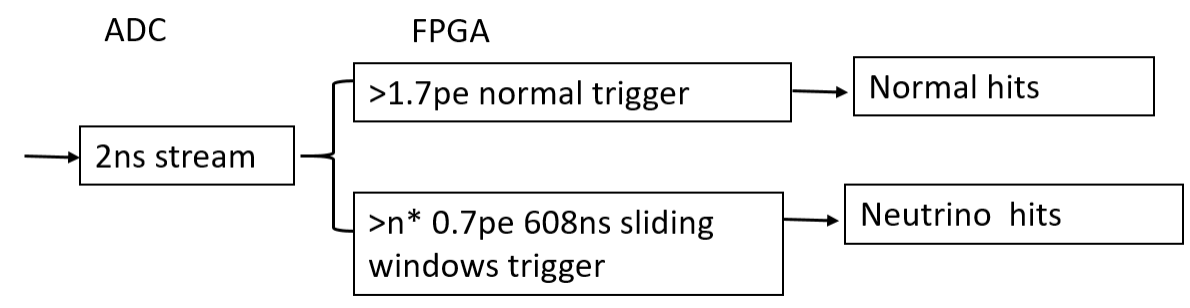

Figure 5: New trigger mode

\section{Significance estimation}

Counting $N_{i}$ pulses during a given time interval $\Delta t$, rates $r_{i}=N_{i} / \Delta t$ for MD i, are derived. The index i ranges from 1 to the total number of MD. $r_{i}$ is rate expectation values approximated by Gaussian distributions, and $\left\langle\sigma_{i}\right\rangle$ is the corresponding standard deviation expectation values. The time windows exclude $30 \mathrm{~s}$ before and after the investigated bin to reduce the impact of a wide signal on the mean rates.

The most likely collective rate deviation $\Delta \mu$ of all single MD noise rates $r_{i}$ from their individual $<r_{i}>$ values, is obtained by maximizing the likelihood

$$
\mathcal{L}(\Delta \mu)=\prod_{i=1}^{N_{M D}} \frac{1}{\sqrt{2 \pi}<\sigma_{i}>} \exp \left(-\frac{\left(r_{i}-\left(<r_{i}>+\epsilon_{i} \Delta \mu\right)\right)^{2}}{2<\sigma_{i}>^{2}}\right.
$$

Here $\epsilon_{i}$ denotes a correction for $M D_{i}$ and detection probabilities.

From the calculation from Super-K experiment[10], LHAASO-MD could have 12750 IBD events within 10 seconds if a nearby supernova bursts. For the first second, each MD unit could have 12750/2/1171=5 IBD events. Now the average noise rate is about $2000 \mathrm{~Hz}$. Significance for 1171 MDs:

$$
\sqrt{1171} \times 5 \div \sqrt{2000}=3.5 \sigma
$$

To get a better significance, it is important to lower the noise level using different methods. The noise from electronics and other random sources could be suppressed by FPGA programming. Those Muon events could be reduced by muon template fit.

\section{Conclusions}

We hope to design a dedicated trigger system in the data acquisition system as a supernova neutrino trigger with LHAASO-MD. Now an FPGA Level programming is needed to trigger the low energy signals. We hope LHAASO could be a member experiment of SNEWS in the future.

\section{Acknowledgment}

This work is supported by the Natural Science Foundation of Shandong Province, China (Grant No.ZR2019MA014), Young Scholars Program of Shandong University (Grant No.2018WLJH78), and National Science Foundation of China (NSFC)(Grant No. 11775131, No. U1931108). 


\section{References}

[1] Zhen, Cao. A future project at tibet: The large high altitude air shower observatory (LHAASO). Chinese Physics C. 34. 249(2010). 10.1088/1674-1137/34/2/018

[2] Janka HT. (2016) Neutrino Emission from Supernovae. In: Alsabti A., Murdin P. (eds) Handbook of Supernovae. Springer, Cham. https://doi.org/10.1007/978-3-319-20794-0_4-1

[3] Hirata K, Kajita T, Koshiba M, Nakahata M, Oyama Y (1987) Observation of a neutrino burst from the supernova SN1987A. Physical Review Letters 58:1490-1493, DOI 10.1103/PhysRevLett.58.1490

[4] Bionta RM, Blewitt G, Bratton CB, Casper D, Ciocio A (1987) Observation of a neutrino burst in coincidence with supernova 1987A in the Large Magellanic Cloud. Physical Review Letters 58:1494-1496, DOI 10.1103/PhysRevLett.58.1494

[5] Alexeyev EN, Alexeyeva LN, Krivosheina IV, Volchenko VI (1988) Detection of the neutrino signal from SN 1987A in the LMC using the INR Baksan underground scintillation telescope. Physics Letters B 205:209-214, DOI 10.1016/0370-2693(88)91651-6

[6] Ando, Shin'ichiro \& Beacom, John \& Yüksel, Hasan. (2005). Detection of Neutrinos from Supernovae in Nearby Galaxies. Physical review letters. 95. 171101. 10.1103/PhysRevLett.95.171101.

[7] Habig, A., Scholberg, K. The Supernova Early Warning System. Nat Rev Phys 2, 458-460 (2020). https://doi.org/10.1038/s42254-020-0221-5

[8] He, H., For the LHAASO Collaboration. Design of the LHAASO detectors. Radiat Detect Technol Methods 2, 7 (2018). https://doi.org/10.1007/s41605-018-0037-3

[9] https://geant4.web.cern.ch

[10] Masa-Toshi Koshiba, Observational Neutrino Astrophysics, Physics Today 40, 12, 38 (1987); https://doi.org/10.1063/1.881092

\section{Full Authors List: LHAASO Collaboration}

Zhen $\mathrm{Cao}^{1,2,3}$, F. Aharonian ${ }^{4,5}$, Q. An ${ }^{6,7}$, Axikegu $^{8}$, L.X. Bai ${ }^{9}$, Y.X. Bai ${ }^{1,3}$, L.X. Bai ${ }^{9}$, Y.X. Bai ${ }^{1,3}$, Y.W. Bao ${ }^{10}$, D. Bastieri ${ }^{11}$, X.J. Bi $1^{1,2,3}$, Y.J. Bi ${ }^{1,3}$, H. Cai ${ }^{12}$, J.T. Cai ${ }^{11}$, Zhe $\mathrm{Cao}^{6,7}$, J. Chang ${ }^{13}$, J.F. Chang ${ }^{1,3,6}$, B.M. Chen ${ }^{14}$, E.S. Chen ${ }^{1,2,3}$, J. Chen ${ }^{9}$, Liang Chen $^{1,2,3}$, Liang Chen ${ }^{15}$, Long Chen ${ }^{8}$, M.J. Chen ${ }^{1,3}$, M.L. Chen ${ }^{1,3,6}$, Q.H. Chen ${ }^{8}$, S.H. Chen ${ }^{1,2,3}$, S.Z. Chen ${ }^{1,3}$, T.L. Chen ${ }^{16}$,X.L. Chen $^{1,2,3}$, Y. Chen ${ }^{10}$, N. Cheng ${ }^{1,3}$, Y.D. Cheng ${ }^{1,3}$, S.W. Cui ${ }^{14}$, X.H. Cui ${ }^{17}$, Y.D. Cui ${ }^{18}$, B. D'Ettorre Piazzoli ${ }^{19}$, B.Z. Dai ${ }^{20}$, H.L. Dai $^{1,3,6}$, Z.G. Dai ${ }^{7}$, Danzengluobu ${ }^{16}$, D. della Volpe ${ }^{21}$, X.J. Dong ${ }^{1,3}$, K.K. Duan ${ }^{13}$, J.H. Fan ${ }^{11}$, Y.Z. Fan ${ }^{13}$, Z.X. Fan ${ }^{1,3}$, J. Fang ${ }^{20}$, K. Fang $^{1,3}$, C.F. Feng ${ }^{22}$, L. Feng ${ }^{13}$, S.H. Feng ${ }^{1,3}$, Y.L. Feng ${ }^{13}$, B. Gao ${ }^{1,3}$, C.D. Gao ${ }^{22}$, L.Q. Gao ${ }^{1,2,3}$, Q. Gao ${ }^{16}$, W. Gao ${ }^{22}$, M.M. Ge ${ }^{20}$, L.S. Geng ${ }^{1,3}$, G.H. Gong ${ }^{23}$, Q.B. Gou ${ }^{1,3}$, M.H. Gu ${ }^{1,3,6}$, F.L. Guo ${ }^{15}$, J.G. Guo ${ }^{1,2,3}$, X.L. Guo ${ }^{8}$, Y.Q. Guo ${ }^{1,3}$, Y.Y. Guo ${ }^{1,2,3,13}$, Y.A. $\mathrm{Han}^{24}$, H.H. He $\mathrm{He}^{1,2,3}$, H.N. He ${ }^{13}$, J.C. He ${ }^{1,2,3}$, S.L. He ${ }^{11}$, X.B. He ${ }^{18}$, Y. He ${ }^{8}$, M. Heller ${ }^{21}$, Y.K. Hor ${ }^{18}$, C. Hou ${ }^{1,3}$, H.B. Hu ${ }^{1,2,3}$, S. $\mathrm{Hu}^{9}$, S.C. $\mathrm{Hu}^{1,2,3}$, X.J. $\mathrm{Hu}^{23}$, D.H. Huang ${ }^{8}$, Q.L. Huang ${ }^{1,3}$, W.H. Huang ${ }^{22}$, X.T. Huang ${ }^{22}$, X.Y. Huang ${ }^{13}$, Z.C. Huang ${ }^{8}$, F. Ji ${ }^{1,3}$, X.L. $\mathrm{Ji}^{1,3,6}$, H.Y. Jia ${ }^{8}$, K. Jiang ${ }^{6,7}$, Z.J. Jiang ${ }^{20}$, C. Jin ${ }^{1,2,3}$, T. Ke ${ }^{1,3}$, D. Kuleshov ${ }^{25}$, K. Levochkin ${ }^{25}$, B.B. Li ${ }^{14}$, Cheng Li ${ }^{6,7}$, Cong Li ${ }^{1,3}$,

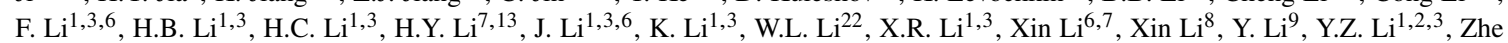

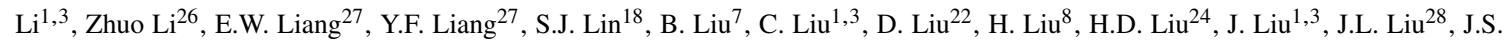

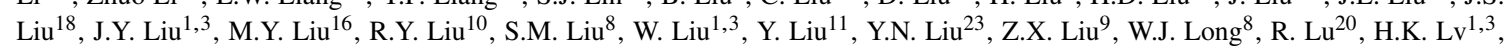




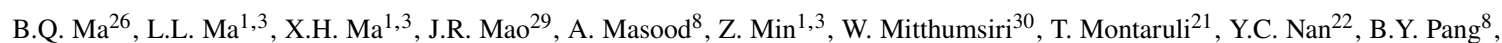
P. Pattarakijwanich ${ }^{30}$, Z.Y. Pei ${ }^{11}$, M.Y. Qi ${ }^{1,3}$, Y.Q. Qi ${ }^{14}$, B.Q. Qiao ${ }^{1,3}$, J.J. Qin ${ }^{7}$, D. Ruffolo ${ }^{30}$, V. Rulev ${ }^{25}$, A. Sáiz ${ }^{30}$, L. Shao ${ }^{14}$, O. Shchegolev $^{25,31}$, X.D. Sheng ${ }^{1,3}$, J.Y. Shi ${ }^{1,3}$, H.C. Song ${ }^{26}$, Yu.V. Stenkin ${ }^{25,31}$, V. Stepanov ${ }^{25}$, Y. Su ${ }^{32}$, Q.N. Sun ${ }^{8}$, X.N. Sun ${ }^{27}$, Z.B.

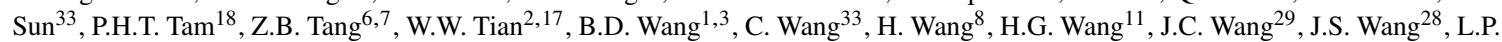
Wang $^{22}$, L.Y. Wang ${ }^{1,3}$, R.N. Wang ${ }^{8}$, W. Wang ${ }^{18}$, W. Wang ${ }^{12}$, X.G. Wang ${ }^{27}$, X.J. Wang ${ }^{1,3}$, X.Y. Wang ${ }^{10}$, Y. Wang ${ }^{8}$, Y.D. Wang ${ }^{1,3}$, Y.J. Wang $^{1,3}$, Y.P. Wang ${ }^{1,2,3}$, Z.H. Wang ${ }^{9}$, Z.X. Wang ${ }^{20}$, Zhen Wang ${ }^{28}$, Zheng Wang ${ }^{1,3,6}$, D.M. Wei ${ }^{13}$, J.J. Wei ${ }^{13}$, Y.J. Wei ${ }^{1,2,3}$, T. Wen ${ }^{20}$, C.Y. $\mathrm{Wu}^{1,3}$, H.R. $\mathrm{Wu}^{1,3}$, S. $\mathrm{Wu}^{1,3}$, W.X. Wu ${ }^{8}$, X.F. Wu ${ }^{13}$, S.Q. Xi ${ }^{1,3}$, J. Xia ${ }^{7,13}$, J.J. Xia ${ }^{8}$, G.M. Xiang ${ }^{2,15}$, D.X. Xiao ${ }^{16}$, G. Xiao ${ }^{1,3}$, H.B. Xiao ${ }^{11}$, G.G. Xin ${ }^{12}$, Y.L. Xin ${ }^{8}$, Y. Xing ${ }^{15}$, D.L. Xu ${ }^{28}$, R.X. Xu ${ }^{26}$, L. Xue ${ }^{22}$, D.H. Yann ${ }^{29}$, J.Z. Yan ${ }^{13}$, C.W. Yang 9 , F.F. Yang ${ }^{1,3,6}$, J.Y. Yang ${ }^{18}$, L.L. Yang ${ }^{18}$, M.J. Yang ${ }^{1,3}$, R.Z. Yang ${ }^{7}$, S.B. Yang ${ }^{20}$, Y.H. Yao ${ }^{9}$, Z.G. Yao ${ }^{1,3}$, Y.M. Ye ${ }^{23}$, L.Q. Yin ${ }^{1,3}$, N. Yin ${ }^{22}$, X.H. You $^{1,3}$, Z.Y. You ${ }^{1,2,3}$, Y.H. Yu ${ }^{22}$, Q. Yuan ${ }^{13}$, H.D. Zeng ${ }^{13}$, T.X. Zeng ${ }^{1,3,6}$, W. Zeng ${ }^{20}$, Z.K. Zeng ${ }^{1,2,3}$, M. Zha ${ }^{1,3}$, X.X. Zhai ${ }^{1,3}$, B.B. Zhang $^{10}$, H.M. Zhang ${ }^{10}$, H.Y. Zhang ${ }^{22}$, J.L. Zhang ${ }^{1}$, J.W. Zhang ${ }^{9}$, L.X. Zhang ${ }^{11}$, Li Zhang ${ }^{20}$, Lu Zhang ${ }^{14}$, P.F. Zhang ${ }^{20}$, P.P. Zhang ${ }^{14}$, R. Zhang ${ }^{7,13}$, S.R. Zhang ${ }^{14}$, S.S. Zhang ${ }^{1,3}$, X. Zhang ${ }^{10}$, X.P. Zhang ${ }^{1,3}$, Y.F. Zhang ${ }^{8}$, Y.L. Zhang ${ }^{1,3}$, Yi Zhang ${ }^{1,13}$, Yong Zhang ${ }^{1,3}$, B. $Z_{\text {Zhao }}^{8}$, J. Zhao ${ }^{1,3}$, L. Zhao ${ }^{6,7}$, L.Z. Zhao ${ }^{14}$, S.P. Zhao ${ }^{13,22}$, F. Zheng ${ }^{33}$, Y. Zheng ${ }^{8}$, B. Zhou ${ }^{1,3}$, H. Zhou ${ }^{28}$, J.N. Zhou ${ }^{15}$, P. Zhou ${ }^{10}$, R. Zhou $^{9}$, X.X. Zhou ${ }^{8}$, C.G. Zhu ${ }^{22}$, F.R. Zhu ${ }^{8}$, H. Zhu ${ }^{17}$, K.J. Zhu ${ }^{1,2,3,6}$ and X. Zuo ${ }^{1,3}$

${ }^{1}$ Key Laboratory of Particle Astrophyics \& Experimental Physics Division \& Computing Center, Institute of High Energy Physics, Chinese Academy of Sciences, 100049 Beijing, China. ${ }^{2}$ University of Chinese Academy of Sciences, 100049 Beijing, China. ${ }^{3}$ TIANFU Cosmic Ray Research Center, Chengdu, Sichuan, China. ${ }^{4}$ Dublin Institute for Advanced Studies, 31 Fitzwilliam Place, 2 Dublin, Ireland. ${ }^{5}$ Max-Planck-Institut for Nuclear Physics, P.O. Box 103980, 69029 Heidelberg, Germany. ${ }^{6}$ State Key Laboratory of Particle Detection and Electronics, China. ${ }^{7}$ University of Science and Technology of China, 230026 Hefei, Anhui, China. ${ }^{8}$ School of Physical Science and Technology \& School of Information Science and Technology, Southwest Jiaotong University, 610031 Chengdu, Sichuan, China. ${ }^{9}$ College of Physics, Sichuan University, 610065 Chengdu, Sichuan, China. ${ }^{10}$ School of Astronomy and Space Science, Nanjing University, 210023 Nanjing, Jiangsu, China. ${ }^{11}$ Center for Astrophysics, Guangzhou University, 510006 Guangzhou, Guangdong, China. ${ }^{12}$ School of Physics and Technology, Wuhan University, 430072 Wuhan, Hubei, China. ${ }^{13}$ Key Laboratory of Dark Matter and Space Astronomy, Purple Mountain Observatory, Chinese Academy of Sciences, 210023 Nanjing, Jiangsu, China. ${ }^{14}$ Hebei Normal University, 050024 Shijiazhuang, Hebei, China. ${ }^{15}$ Key Laboratory for Research in Galaxies and Cosmology, Shanghai Astronomical Observatory, Chinese Academy of Sciences, 200030 Shanghai, China. ${ }^{16} \mathrm{Key}$ Laboratory of Cosmic Rays (Tibet University), Ministry of Education, 850000 Lhasa, Tibet, China. ${ }^{17}$ National Astronomical Observatories, Chinese Academy of Sciences, 100101 Beijing, China. ${ }^{18}$ School of Physics and Astronomy \& School of Physics (Guangzhou), Sun Yat-sen University, 519000 Zhuhai, Guangdong, China. ${ }^{19}$ Dipartimento di Fisica dell’Università di Napoli ‘Federico II”, Complesso Universitario di Monte Sant'Angelo, via Cinthia, 80126 Napoli, Italy. ${ }^{20}$ School of Physics and Astronomy, Yunnan University, 650091 Kunming, Yunnan, China. ${ }^{21}$ D'epartement de Physique Nucl'eaire et Corpusculaire, Facult'e de Sciences, Universit'e de Gen'eve, 24 Quai Ernest Ansermet, 1211 Geneva, Switzerland. ${ }^{22}$ Institute of Frontier and Interdisciplinary Science, Shandong University, 266237 Qingdao, Shandong, China. ${ }^{23}$ Department of Engineering Physics, Tsinghua University, 100084 Beijing, China. ${ }^{24}$ School of Physics and Microelectronics, Zhengzhou University, 450001 Zhengzhou, Henan, China. ${ }^{25}$ Institute for Nuclear Research of Russian Academy of Sciences, 117312 Moscow, Russia. ${ }^{26}$ School of Physics, Peking University, 100871 Beijing, China. ${ }^{27}$ School of Physical Science and Technology, Guangxi University, 530004 Nanning, Guangxi, China. ${ }^{28}$ Tsung-Dao Lee Institute \& School of Physics and Astronomy, Shanghai Jiao Tong University, 200240 Shanghai, China. ${ }^{29}$ Yunnan Observatories, Chinese Academy of Sciences, 650216 Kunming, Yunnan, China. ${ }^{30}$ Department of Physics, Faculty of Science, Mahidol University, 10400 Bangkok, Thailand. ${ }^{31}$ Moscow Institute of Physics and Technology, 141700 Moscow, Russia. ${ }^{32}$ Key Laboratory of Radio Astronomy, Purple Mountain Observatory, Chinese Academy of Sciences, 210023 Nanjing, Jiangsu, China. ${ }^{33}$ National Space Science Center, Chinese Academy of Sciences, 100190 Beijing, China. 